\title{
Cardiac Chloroma Clinically Resembling Pulmonary Embolism in the Emergency Setting
}

\author{
Albert Pons Escoda, Diego Nova Vaca, Lucía Aja Rodríguez, \\ Matías De Albert de Delás Vigo, Fernando Fernández Alarza \\ Department of Radiology, Hospital Universitari de Bellvitge, L'Hospitalet de Llobregat, Spain \\ Email: aponse@hotmail.com
}

Received 26 January 2014; revised 2 March 2014; accepted 10 March 2014

Copyright (C) 2014 by authors and Scientific Research Publishing Inc.

This work is licensed under the Creative Commons Attribution International License (CC BY). http://creativecommons.org/licenses/by/4.0/

(c) (i) Open Access

\begin{abstract}
We report the case of a 52-year-old man with a history of acute myeloid leukemia who presented to the emergency room with fatigue, dyspnea and chest pain. Initial routine examination showed signs of right heart failure. A CT was requested to rule out pulmonary embolism. The imaging revealed the presence of a cardiac infiltrative mass involving the heart's right free wall that proved to be a chloroma.
\end{abstract}

\section{Keywords}

Acute Myelogenous Leukemia; Chloroma; Granulocytic Sarcoma; Heart

\section{Introduction}

We report the case of a 52-year-old man with a history of acute myeloid leukemia (AML) who presented to the emergency room with fatigue, dyspnea and chest pain. The imaging revealed the presence of a cardiac infiltrative mass involving the heart's right free wall that proved to be a chloroma. We present the clinical scenario, the imaging findings and a brief review of the literature regarding this rare disease and specially its exceptional location.

\section{Case Report}

We present the case of a 52-year-old man with a history of AML, which diagnosis was based on hematologic and bone marrow analysis approximately 3 years ago, and that was treated with allogenic progenitor cell transplantation. 
The patient presented to the emergency room with fatigue, dyspnea and discrete chest pain during the last days.

The initial routine laboratory test showed no significant abnormality. An electrocardiography (ECG) revealed subtle signs of right heart failure. Consequently a chest CT angiogram was requested to rule out pulmonary embolism (PE).

CT examination showed a large, well-marginated, lobulated, diffuse myocardial thickening involving the free walls of the right cardiac chambers. It encased the right coronary artery without narrowing its lumen and compressed the right ventricle. In the pulmonary angiographic phase, obtained $20 \mathrm{~s}$ after contrast administration, the mass-like myocardial thickening had no or slight contrast uptake. Subsequently, the on call radiologist decided to obtain a second delayed phase to evaluate the pattern of enhancement. It was acquired $120 \mathrm{~s}$ after contrast administration and it showed a progressive, slow enhancement similar to that of the myocardium. Some hypodense areas could be seen, probably due to necrosis or wash-out. The latter finding highly suggested the suspected tumoral nature of the myocardial thickening (Figure 1).

The CT findings resembled those of a lymphoproliferative or myeloproliferative disorder involving the myocardium. Pursuant to such specific clinical scenario, the diagnostic of heart chloroma (granulocytic sarcoma) was suggested.

A further revision of the images discovered a subcutaneous solid nodule in the chest wall.

The following day, the patient underwent a biopsy of the subcutaneous nodule.

The specimen demonstrated diffuse and infiltrative proliferation of undifferentiated mononuclear malignant cells characterized by granular cytoplasm consistent with myelocytic differentiation. Immunohistochemistry was positive for myeloperoxidase.

These findings confirmed the extramedullary leukemic recurrence and the cardiac mass were labeled as a cardiac chloroma.
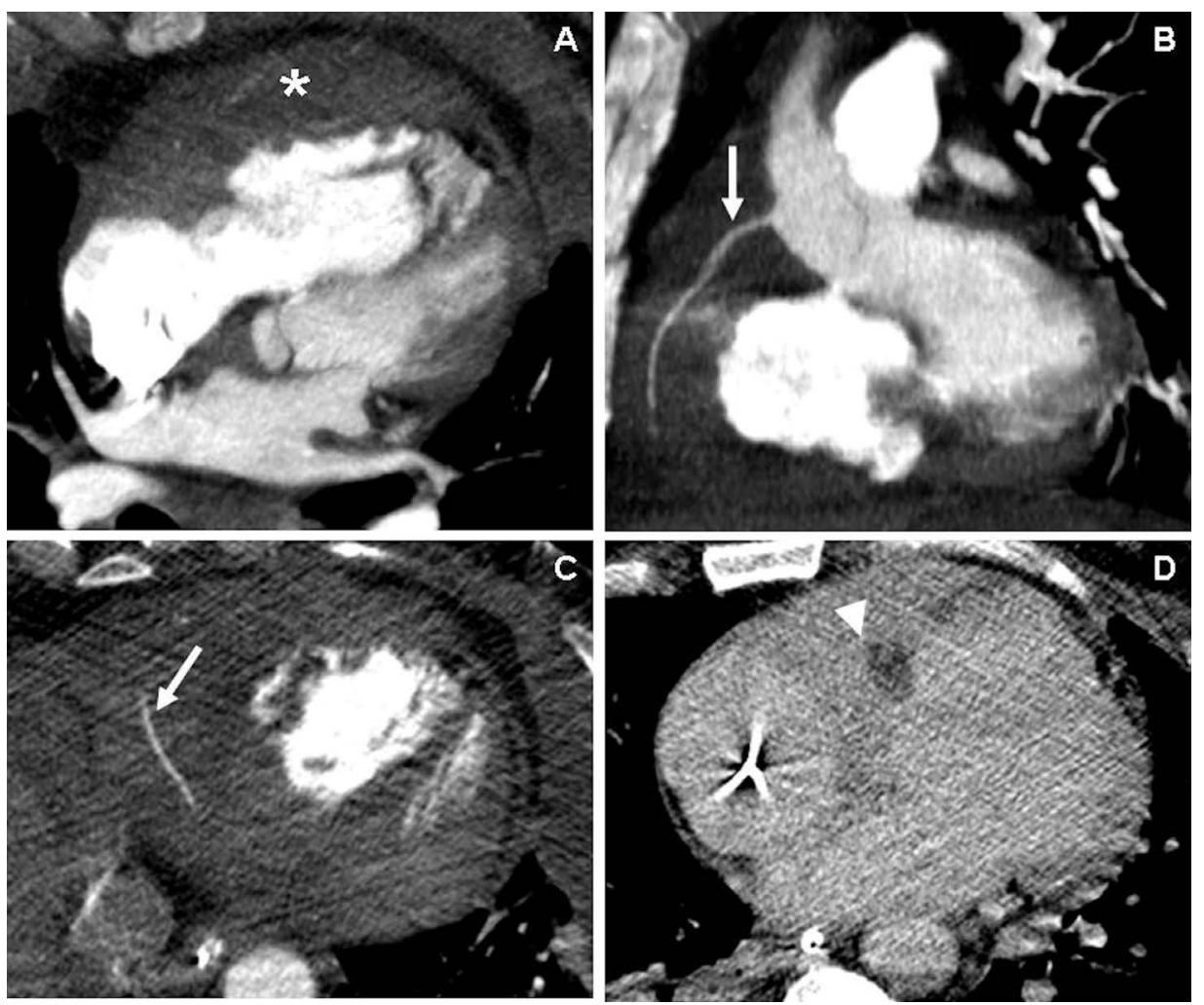

Figure 1. Chest CT angiogram on 16-MDCT (GE Medical Systems, Bright Speed. 100 kv. 250 $\mathrm{mA}$. Slice thickness $1.25 \mathrm{~mm}$. Contrast agent: Iodixanol $320 \mathrm{mg} / \mathrm{ml}, 80 \mathrm{ml}, 5 \mathrm{ml} / \mathrm{s}$ ). 52 year-old man. Cardiac Chloroma. Large, well-marginated, lobulated, diffuse myocardial thickening involving the free walls of the right cardiac chambers (asterisk in A). It encases the right coronary artery without narrowing its lumen (arrows in B and C). A delayed phase $120 \mathrm{~s}$ after contrast administration showed areas of necrosis or wash-out (dashed arrow in D). 
During the weeks following, the patient developed severe acute renal failure and died in the setting of multiorganic failure.

\section{Discussion}

Chloroma, also known as granulocytic sarcoma, is a localized extramedullary tumor composed of malignant cells of the myeloid cell line, most frequently occurring in myelogenous leukemia [1].

Granulocytic sarcomas have been observed in patients with acute myelogenous leukemia, chronic myelogenous leukemia, and other myeloproliferative disorders such as myelofibrosis with myeloid metaplasia, hypereosinophilic syndrome, and polycythemia vera. They occur in $2.5 \%-9.1 \%$ of patients with acute myelogenous leukemia and five times less frequently in patients with chronic myelogenous leukemia. There is no age or sex predilection associated with this tumor [2].

This tumor can affect almost any organ in the body, the most common sites of involvement being bone, soft tissue, lymph nodes, and skin. The diagnosis is always challenging and is ultimately made using histochemical and pathological methods [1] [2].

Heart involvement is exceptional; only about 20 cases have been reported in the reviewed literature. It can present as a diffuse myocardial widening or less frequently as a focal infiltrative mass. All layers, chambers, and valves of the heart may be involved but a natural tendency to involve the right ventricle and atrium has been reported [1]-[4]. This tendency explains a possible clinical presentation as right heart failure as seen in our case [3]-[5].

In the reviewed literature, CT usually shows diffuse widening of the right atrium and ventricle walls, with little or no contrast enhancement. Furthermore, the coronary arteries can be seen across the infiltrative tissue without narrowing of its lumen, a characteristic radiological feature for lymphoproliferative and myeloproliferative disorders [1]-[4] [6]. The latter feature is nicely depicted in the images of our case and it was the main CT finding that raised the diagnostic suspicion.

Granulocytic sarcoma of the heart requires early diagnosis because a focal relapse can eventually be solved both by local and systemic therapy, leading to shrinkage of the mass and normalization of the affected myocardium [5].

Awareness of potential sites of involvement, morphologic and imaging features could help radiologists to consider granulocytic sarcoma within a differential diagnosis in the proper clinical setting [1].

\section{References}

[1] Fritz, J., Vogel, W., Bares, R. and Horger, M. (2007) Radiologic Spectrum of Extramedullary Relapse of Myelogenous Leukemia in Adults. American Journal of Roentgenology, 189, 209-218. http://dx.doi.org/10.2214/AJR.06.1500

[2] Guermazi, A., Feger, C., Rousselot, P., Merad, M., Benchaib, N., Bourrier, P., et al. (2002) Granulocytic Sarcoma (Chloroma): Imaging Findings in Adults and Children. American Journal of Roentgenology, 178, 319-325. http://dx.doi.org/10.2214/ajr.178.2.1780319

[3] Attallah, A., Cheong, B.Y., Bernicker, E. and Wilson, J.M. (2010) Cardiac Chloroma: Novel Presentation and Subsequent Diagnosis with Cardiac Magnetic Resonance Imaging. Texas Heart Institute Journal, 37, 242-243.

[4] Tsai, J. and Lee, E. (2010) MDCT Imaging Findings of Extramedullary Granulocytic Sarcoma of the Heart. Journal of Thoracic Imaging, 25, W14-16. http://dx.doi.org/10.1097/RTI.0b013e3181a6d0eb

[5] Mawad, R., Wu, D., Abkowitz, J.L. and Walter, R.B. (2012) Myeloid Sarcoma of the Heart. Leukemia \& Lymphoma, 53, 2511-2514. http://dx.doi.org/10.3109/10428194.2012.685736

[6] Jeudy, J., Kirsch, J., Tavora, F., Burke, A., Franks, T., Mohammed, T.L., et al. (2012) Cardiac Lymphoma: RadiologicPathologic Correlation. RadioGraphics, 32, 1369-1380. http://dx.doi.org/10.1148/rg.325115126

\section{Abbreviations}

AML $=$ acute myeloid leukemia.

$\mathrm{CT}=$ computed tomography.

ECG = electrocardiography.

$\mathrm{PE}=$ pulmonary embolism. 